Research Paper

\title{
Epithelial-Mesenchymal Transition is Superior to Vessels-Encapsulate Tumor Cluster in Promoting Metastasis of Hepatocellular Carcinoma: a Morphological Evidence
}

\author{
Chuanchao He ${ }^{1,2^{*}}$, Zhenyu Zhou ${ }^{1,2^{*}}$, Hai Jiang ${ }^{1,2^{*}}, Z_{i} Y_{i n}^{3}$, Shiyu Meng ${ }^{1,4}$, Jianlong Zhang 1,2 , Pinbo Huang ${ }^{1,2}$ \\ Kang $X_{u^{1,2}}$, Lijuan Bian ${ }^{1,5 凶}$, Zhiyu Xiao ${ }^{1,2 \bowtie}$, Jie Wang ${ }^{1,2 \bowtie}$ \\ 1. Guangdong Province Key Laboratory of Malignant Tumor Epigenetics and Gene Regulation, Research Center of Medicine, Sun Yat-Sen Memorial Hospital, No. 107 \\ Yanjiang Western Road, Guangzhou 510120, China. \\ 2. Department of Hepatobiliary Surgery, Sun Yat-Sen Memorial Hospital, No. 33 Yingfeng Road, Guangzhou 510289, China. \\ 3. General Surgery Department, Guangdong General Hospital, Guangdong Academy of Medical Sciences, No. 106 Zhongshan Er Road., Guangzhou, 510080, China. \\ 4. Department of Anesthesiology, Sun Yat-Sen Memorial Hospital, No. 33 Yingfeng Road, Guangzhou 510289, China. \\ 5. Department of Pathology, Sun Yat-Sen Memorial Hospital, No. 107 Yanjiang Western Road, Guangzhou 510120, China. \\ *These authors contributed equally to this work.
}

$\triangle$ Corresponding authors: Department of Pathology, Sun Yat-Sen Memorial Hospital, No. 107 Yanjiang Western Road, Guangzhou 510120, China. Tel./fax: +86 20 81332781. E-mail: Lijuanbian2004@163.com. Department of Hepatobiliary Surgery, Sun Yat-Sen Memorial Hospital, No. 33 Yingfeng Road, Guangzhou 510289, China. Tel./fax: +86 20 34071073. E-mail: jiewsysu@163.com (Jie Wang), xzysurgeon@hotmail.com (Zhiyu Xiao).

(C) Ivyspring International Publisher. This is an open access article distributed under the terms of the Creative Commons Attribution (CC BY-NC) license (https://creativecommons.org/licenses/by-nc/4.0/). See http://ivyspring.com/terms for full terms and conditions.

Received: 2016.07.05; Accepted: 2016.09.18; Published: 2017.01.01

\begin{abstract}
Purpose Vessels-encapsulate tumor cluster (VETC) is a vascular pattern distinct from classical capillary-like pattern. It is reported that VETC structure is common in hepatocellular carcinoma (HCC) and can promote HCC metastasis in an epithelial-mesenchymal transition (EMT)-independent but VETC-dependent manner. However, the main metastatic manner of HCC containing both VETC and classical vascular structure (we called VETC \pm ) is unknown.

Methods Vascular pattern types and E-cadherin expression were evaluated by immunohistochemical staining in $168 \mathrm{HCC}$ tissues, 50 pairs of primary HCC tissues and intrahepatic metastatic lesions, as well as 12 pairs of primary HCC tissues and major portal vein tumor thrombus. Survival and recurrence rates were evaluated using Kaplan-Meier analysis. The multivariate Cox proportional hazards model was used to determine the independent prognostic factors of $\mathrm{HCC}$.

Results VETC ${ }^{ \pm}$cases were more common than VETC ${ }^{+}$cases $(\mathrm{HCC}$ tissues with a VETC pattern fully distributed in the HCC section) in HCC. Statistical analysis showed that VETC \pm was an independent predictor of survival and recurrence. Furthermore, E-cadherin was positively correlated with the presence of VETC structure. In the case of HCCs with VETC ${ }^{ \pm}$, their metastases (both intrahepatic and major vascular) were more likely to be VETC negative.
\end{abstract}

Conclusions Our findings suggest that EMT may be superior to VETC in promoting HCC metastasis. Thus, both anti-EMT and anti-VETC agents should be considered in the case of HCC with VETC

Key words: hepatocellular carcinoma, epithelial-mesenchymal transition, vessels-encapsulate tumor cluster, angiogenesis, prognosis.

\section{Introduction}

Ranked as the sixth most lethal malignancy, hepatocellular carcinoma (HCC) draws great attention because of its large patient population, aggressive behavior, and low response rates to treatments [1]. Although surgical resection is considered as a potentially curative treatment for 
HCC patients, it is only amenable to early-stage HCC patients but can rarely reverse the poor prognosis of advanced HCC patients [2,3]. Tumor metastasis is the major reason for poor prognosis of HCC patients after hepatic resection [4]. Thus, understanding the mechanisms of HCC metastasis will improve HCC treatment outcomes.

Tumor metastasis is a multistep process which can be briefly divided into invasion and metastasis [5]. Concretely, for invasion, tumor cells should experience a succession of cell-biologic changes, such as local invasion and intravasation into nearby vessels [6]. Although many other modes of invasion have been reported, epithelial-mesenchymal transition (EMT) is still considered to play a key role in these malignant activities [7, 8]. During EMT, tumor cells lose epithelial characteristics and transform into mesenchymal cells. The transformed cells then acquire multiple attributes that enable tumor cells to invade and metastasize [9].

Intravasation is a process depending on the access of tumor cells to vessels, and thus tumors with more vessels would be expected to have a higher intravasation rate [10]. Actually, tumor-associated vasculature has been proved to correlate with tumor metastasis [11, 12]. Interestingly, recent studies reported a new vascular pattern type, called vessels-encapsulate tumor cluster (VETC), is associated with HCC metastasis [13-16]. The new vascular pattern, which is distinct from classical capillary-like vessels, forms a cobweb-like network and envelops HCC cells to form tumor islands [15]. Evidences showed that VETC is a common vascular pattern which promotes HCC metastasis in an EMT-independent but VETC-dependent manner [15]. These observations prompted us to consider which metastatic mode plays a major role during HCC metastasis if the tissue containing both VETC and classical vascular structure, EMT or VETC?

In this study, we used tissue microarrays containing 168 HCC samples of our center to explore the frequency of vascular pattern type in HCC. Besides, we collected 50 pairs of primary HCC tissues and intrahepatic metastatic lesions, as well as 12 pairs of primary HCC tissues and major portal vein tumor thrombi (mPVTTs) to explore the major metastatic manner of HCC. We find that HCC tissues containing both VETC and capillary-like structure are more common than those with a VETC pattern in a whole HCC section. HCC containing this mixed vascular pattern is significantly associated with poor prognosis. Moreover, we provide Morphological evidence that HCC with this mixed structure may probably metastasize via an EMT-dependent manner.

\section{Methods}

\section{Patients and Follow-up}

In this study, the diagnosis of HCC was based on pathology or dynamic contrast-enhanced imaging (computed tomography scan or magnetic resonance imaging) according to the diagnostic criteria of the European Association for the Study of the Liver (EASL, 2012). The surgical indications for HCC were: (1) patients with Child-Pugh class A or indocyanine green at $15 \mathrm{~min}$ (ICG15) $\leq 10 \%$, (2) tumors were resectable with adequate future liver volume, (3) without extrahepatic metastases. A total of 168 adult patients with HCC who underwent curative resection between 2006 and 2010 at the Sun Yat-Sen Memorial Hospital were enrolled in this study. The inclusion criteria of the patients were: (1) distinctive pathologic diagnosis of HCC, (2) no anti-cancer treatment before hepatic resection, (3) suitable formalin-fixed, paraffin-embedded tissues, and (4) complete clinicopathologic and follow-up data. The detailed clinical characteristics of the 168 patients are summarized in Table 1. The TNM classification for HCC was based on The American Joint Committee on Cancer/TNM Staging for Liver Tumors (7th edition, 2010).

The median follow-up of this cohort was 37.7 months (range, 1-115 months). Patients were checked every three to six months during the first two years after operations, then every six to twelve months. Each time, patients were monitored by examining serum AFP levels and performing abdominal ultrasound. Magnetic resonance imaging or computed tomography, together with chest radiographic examination, was performed every three to six months, then every six to twelve months. Overall survival and time to recurrence were considered as the primary endpoints. Overall survival was defined as the interval between surgery and death or between surgery and the last follow-up time for surviving patients. The time to recurrence was defined as the interval between surgery and recurrence or between surgery and the last follow-up time for patients without recurrence.

In addition, a total of 50 pairs of primary HCC tissues and intrahepatic metastatic lesions, as well as 12 pairs of primary HCC tissues and mPVTTs were collected after surgical resection. The tissues were formalin-fixed and paraffin-embedded for further immunohistochemistry. In this study, the criteria to define intrahepatic metastasis were: (1) tumors obviously growing from PVTT, (2) multiple satellite nodules which are smaller than the main tumor they surround, and (3) small single tumor which is present near the main tumor with similar histology $[17,18]$. 
The study protocol was approved by the research ethics committee of Sun Yat-Sen University. A written informed consent was obtained from each patient.

\section{Construction of tissue microarrays and immunohistochemistry}

One hundred sixty-eight HCC samples were used for the construction of tissue microarrays as described previously [19]. Briefly, duplicate cylindrical tissue samples of $1 \mathrm{~mm}$ diameter were punched from representative tumor areas of donor blocks. Immunochemical staining was performed according to the manufacturer's protocols as described previously [20]. Antibodies used for immunochemical staining were CD34 (clone QBEnd/10, 1: 200; Santa Cruz Biotechnology), E-cadherin (clone 24E10, 1: 400; Cell Signaling Technology), and Snail (PAS-11923, 1: 100; Thermo Scientific).

\section{Immunohistochemical evaluation}

All slides of immunochemical staining were evaluated independently by two experienced pathologists. The VETC structure was distinguished by the presence of vessels that formed cobweb-like networks and enveloped HCC cells to form tumor islands [13]. The staining result for E-cadherin and snail was semi-quantitatively represented by an immunohistochemical score combined with the intensity and extent of staining. The intensity of staining was scored as 0 (negative), 1 (weak), 2 (strong). The extent of staining was scored as 0 (negative), 1 (1\%-20\%), 2 (21\%-50\%), 3 (51\%-75\%), and 4 (76\%-100\%). The total score was calculated by multiplying the staining intensity and the staining extent. Each case was finally considered negative if the total score was 0 to 1 and positive if the total score was over 2 .

\section{Statistical Analysis}

Statistical analysis was performed with SPSS software (version 17.0). Cumulative survival and recurrence rates were calculated by the Kaplan-Meier analysis and the log-rank test. Based on the variables selected on univariate analysis, the multivariate Cox proportional hazards model was used to determine the independent prognostic factors of HCC. Quantitative data and categorical data were analyzed by the Student's $t$ test and Fisher's exact test, respectively. $P<0.05$ was considered statistically significant.

\section{Results}

\section{The vascular pattern types in HCC}

To explore the vascular pattern types in the HCC cohort of our center, we assessed the immunohistochemical staining for CD34 in tissue microarrays of 168 HCC samples. VETC structure was commonly found in our cohort $(26.2 \%)$. However, we found that in most of VETC-present HCC tissues, the VETC structure was distributed unevenly with classical capillary-like pattern (Fig. 1C).

In order to explore whether this mixed vascular pattern was an important factor in determining clinical outcomes of HCC patients, we evaluated this vascular pattern in 168 HCC samples. We designated the cases with a VETC pattern fully distributed in the HCC section as $\mathrm{VETC}^{+}$, the cases with mixed vascular pattern as VETC ${ }^{ \pm}$, and those without a VETC pattern in a whole HCC section as VETC-. Among 168 HCC tissues examined, $73.8 \%$ were VETC- cases $(124 / 168)$ (Fig. 1A), 20.2\% were VETC ${ }^{ \pm}$cases $(34 / 168)$ (Fig. 1C), and only $6 \%$ were VETC $^{+}$cases (10/168) (Fig. 1B). Correlation analysis demonstrated that these vascular pattern types were significantly associated with vascular invasion, tumor encapsulation, tumor size, tumor differentiation, and TNM stage $(\mathrm{P}<0.05)$ (Table 1).

Table 1. Correlation of vascular pattern types with patient's clinicopathologic features in HCCs.

\begin{tabular}{|c|c|c|c|c|c|c|}
\hline \multirow{2}{*}{\multicolumn{2}{|c|}{$\begin{array}{l}\text { Clinicopathological } \\
\text { Variables }\end{array}$}} & \multirow{2}{*}{$\begin{array}{l}\text { Case } \\
\text { Number } \\
(n=168)\end{array}$} & \multicolumn{3}{|c|}{ Vascular Pattern Types } & \multirow{2}{*}{$\begin{array}{l}P \\
- \text { Value }\end{array}$} \\
\hline & & & $\begin{array}{l}\text { VETC- } \\
(\mathrm{n}=124)\end{array}$ & $\begin{array}{l}\text { VETC+ } \\
(n=10)\end{array}$ & $\begin{array}{l}\text { VETC } \pm \\
(\mathrm{n}=34)\end{array}$ & \\
\hline Age & & & $51.15 \pm 12.8$ & $49.80 \pm 14.4$ & $51.21 \pm 13.1$ & 0.661 \\
\hline \multirow[t]{2}{*}{ Sex } & Female & 32 & 24 & 3 & 5 & \multirow[t]{2}{*}{0.526} \\
\hline & male & 136 & 100 & 7 & 29 & \\
\hline \multirow[t]{2}{*}{ Serum AFP } & $\leq 20 \mathrm{ng} / \mathrm{mL}$ & 43 & 31 & 2 & 8 & \multirow[t]{2}{*}{$>0.999$} \\
\hline & $>20 \mathrm{ng} / \mathrm{mL}$ & 125 & 91 & 8 & 26 & \\
\hline \multirow[t]{2}{*}{ HBsAg } & Negative & 19 & 13 & 2 & 4 & \multirow[t]{2}{*}{0.507} \\
\hline & Positive & 149 & 109 & 8 & 28 & \\
\hline \multirow[t]{2}{*}{ Cirrhosis } & Absent & 40 & 29 & 4 & 7 & \multirow[t]{2}{*}{0.442} \\
\hline & Present & 128 & 95 & 6 & 27 & \\
\hline \multirow[t]{2}{*}{ Tumor size } & $\leq 5 \mathrm{~cm}$ & 70 & 59 & 2 & 9 & \multirow[t]{2}{*}{$0.030^{*}$} \\
\hline & $>5 \mathrm{~cm}$ & 98 & 65 & 8 & 25 & \\
\hline \multirow{2}{*}{$\begin{array}{l}\text { Vascular } \\
\text { invasion }\end{array}$} & Absent & 117 & 93 & 6 & 18 & \multirow[t]{2}{*}{$0.031^{*}$} \\
\hline & Present & 51 & 31 & 4 & 16 & \\
\hline \multirow{2}{*}{$\begin{array}{l}\text { Tumor } \\
\text { encapsulation }\end{array}$} & Absent & 78 & 50 & 6 & 22 & \multirow[t]{2}{*}{$0.028^{*}$} \\
\hline & Present & 90 & 74 & 4 & 12 & \\
\hline \multirow{2}{*}{$\begin{array}{l}\text { Tumor } \\
\text { differentiation }\end{array}$} & I-II & 110 & 91 & 6 & 13 & \multirow[t]{2}{*}{$0.001^{*}$} \\
\hline & III-IV & 58 & 33 & 4 & 21 & \\
\hline \multirow[t]{2}{*}{ TNM stage } & I-II & 63 & 54 & 2 & 7 & \multirow[t]{2}{*}{$0.028^{*}$} \\
\hline & III-IV & 105 & 70 & 8 & 27 & \\
\hline
\end{tabular}

Abbreviations: AFP, a-fetoprotein; HBsAg, hepatitis B surface antigen; TNM, tumor-lymph node-metastasis. ${ }^{*} \mathrm{P}<0.05$. 

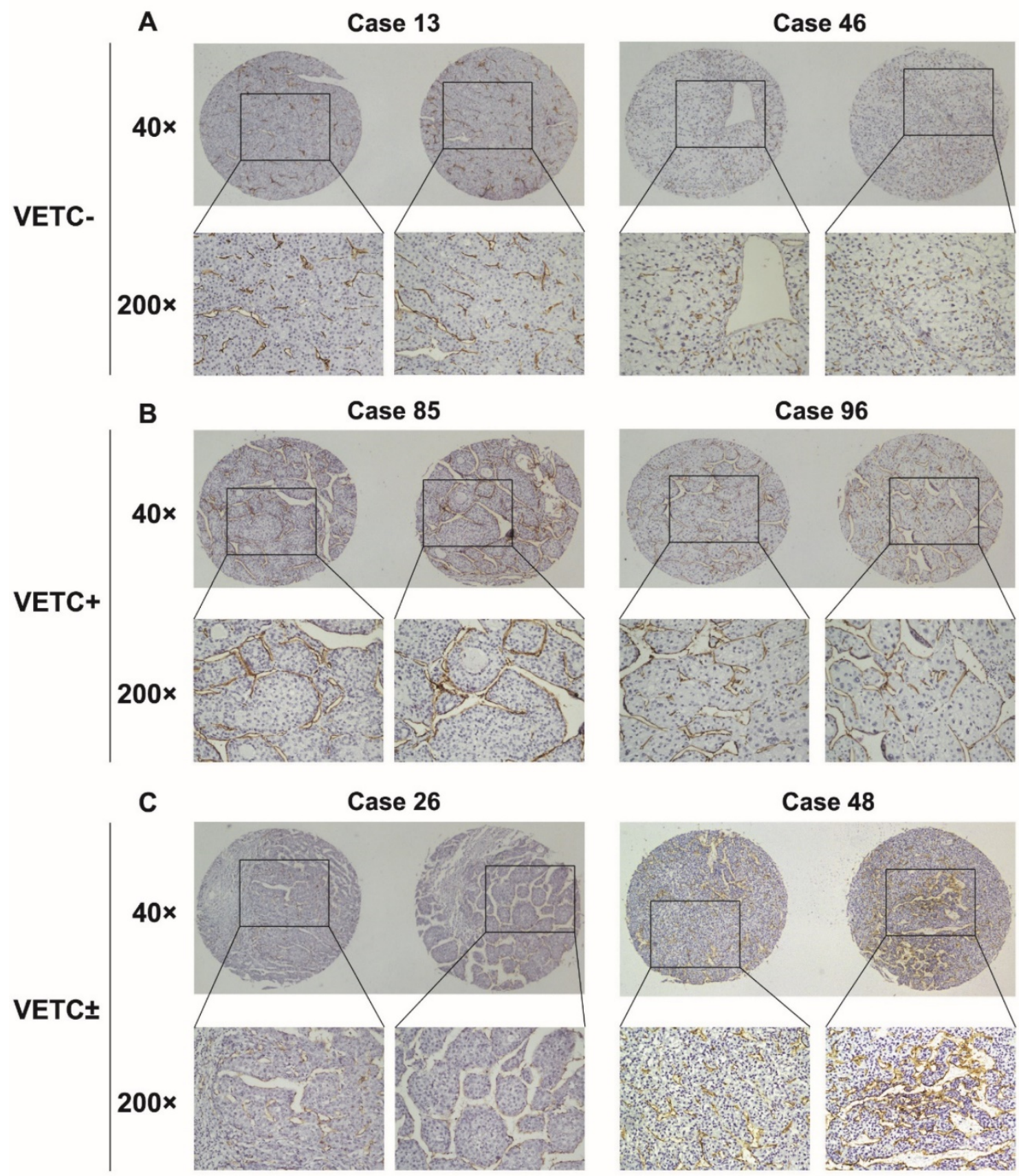

Figure 1. Vascular pattern types in $168 \mathrm{HCC}$ tissues assessed by immunohistochemical staining for CD34. (A) HCC tissues from two different patients have abundant capillary-like vessels but have absolutely no VETCs, which were classified as VETC-. (B) HCC tissues from two different patients showed distribution of VETC structure in a whole HCC section, which were classified as VETC ${ }^{+}$. (C) HCC tissues from two different patients displayed mixed vascular structure containing both VETC and capillary-like structure, which were classified as VETC \pm .

\section{Prognostic role of VETC \pm}

To evaluate prognostic value of $\mathrm{VETC}^{ \pm}$in $\mathrm{HCC}$, we first performed univariate survival analysis. The presence of $\mathrm{VETC}^{ \pm}$, tumor size, vascular invasion, tumor differentiation, and TNM stage were associated with overall survival and recurrence rates (Table 2). Kaplan-Meier analysis revealed HCC patients with VETC $^{ \pm}$had shorter overall survival and higher recurrence rates compared with VETC- group (Fig. 2B) and VETC $^{+}$group (Fig. 2C), respectively. Furthermore, Cox's multivariate proportional hazards model indicated that the presence of $\mathrm{VETC}^{ \pm}$was an independent predictor of survival $(P=0.023)$ and recurrence $(P=0.046)$ in HCC after curative resection (Table 2). 
Table 2. Univerate and multivariate analysis of factors associated with survival and recurrence of $168 \mathrm{HCCs}$.

\begin{tabular}{|c|c|c|c|c|c|c|c|c|c|c|c|c|}
\hline & \multicolumn{3}{|c|}{ Univariate Analysis } & \multicolumn{3}{|c|}{ Multivariate Analysis } & \multicolumn{3}{|c|}{ Univariate Analysis } & \multicolumn{3}{|c|}{ Multivariate Analysis } \\
\hline & HR & $95 \% \mathrm{CI}$ & $P$ value & HR & $95 \% \mathrm{CI}$ & $P$ value & HR & $95 \% \mathrm{CI}$ & $\mathrm{P}$ value & HR & $95 \% \mathrm{CI}$ & $\mathrm{P}$ value \\
\hline $\begin{array}{l}\text { Age } \\
(\leq 49.0 \text { vs. }>49.0)\end{array}$ & 0.743 & $0.490-1.125$ & 0.161 & & & & 0.951 & $0.602-1.322$ & 0.534 & & & \\
\hline $\begin{array}{l}\text { Sex } \\
\text { (female vs. male) }\end{array}$ & 0.864 & $0.499-1.496$ & 0.602 & & & & 1.503 & $0.891-2.537$ & 0.127 & & & \\
\hline $\begin{array}{l}\text { Serum AFP } \\
(\leq 20 \text { vs. }>20 \mathrm{ng} / \mathrm{mL})\end{array}$ & 1.342 & $0.869-2.074$ & 0.185 & & & & 1.383 & $0.926-2.065$ & 0.113 & & & \\
\hline $\begin{array}{l}\text { HBsAg } \\
\text { (negative vs. positive) }\end{array}$ & 0.857 & $0.496-1.483$ & 0.582 & & & & 0.799 & $0.470-1.356$ & 0.405 & & & \\
\hline $\begin{array}{l}\text { Tumor encapsulation } \\
\text { (absent vs. present) }\end{array}$ & 1.453 & $0.985-2.142$ & 0.060 & & & & 1.537 & $1.065-2.192$ & $0.022^{*}$ & & & \\
\hline $\begin{array}{l}\text { Cirrhosis } \\
\text { (absent vs. present) }\end{array}$ & 2.013 & $1.179-3.435$ & $0.010^{*}$ & 2.134 & $1.231-3.701$ & $0.007^{*}$ & 1.011 & $0.658-1.552$ & 0.961 & & & \\
\hline $\begin{array}{l}\text { Tumor size } \\
(\leq 5 \mathrm{vs} .>5 \mathrm{~cm})\end{array}$ & 1.689 & $1.131-2.522$ & $0.010^{*}$ & & & & 2.266 & $1.534-3.348$ & $<0.001^{*}$ & & & \\
\hline $\begin{array}{l}\text { Vascular invasion } \\
\text { (absent vs. present) }\end{array}$ & 2.355 & $1.579-3.513$ & $<0.001^{*}$ & & & & 2.206 & $1.471-3.308$ & $<0.001^{*}$ & & & \\
\hline $\begin{array}{l}\text { Tumor differentiation } \\
\text { (I-II vs. III-IV) }\end{array}$ & 2.318 & $1.538-3.494$ & $<0.001^{*}$ & 1.546 & $1.107-2.159$ & $0.011^{*}$ & 2.046 & $1.522-2.751$ & $<0.001^{*}$ & 1.592 & $1.141-2.222$ & $0.006^{*}$ \\
\hline $\begin{array}{l}\text { TNM stage } \\
\text { (I-II vs. III-IV) }\end{array}$ & 2.056 & $1.349-3.133$ & $0.001^{*}$ & & & & 2.734 & $1.823-4.100$ & $<0.001^{*}$ & 2.492 & $1.016-6.117$ & $0.046^{*}$ \\
\hline $\begin{array}{l}\text { Vascular pattern types } \\
\left(\text { VETC }^{-}, \text {VETC }^{+} \text {vs. VETC }\right. \\
\end{array}$ & 2.181 & $1.409-3.377$ & $0.001^{*}$ & 1.674 & $1.072-2.612$ & $0.023^{*}$ & 2.563 & $1.659-3.961$ & $<0.001^{*}$ & 1.625 & $1.009-2.617$ & $0.046^{*}$ \\
\hline
\end{tabular}

A
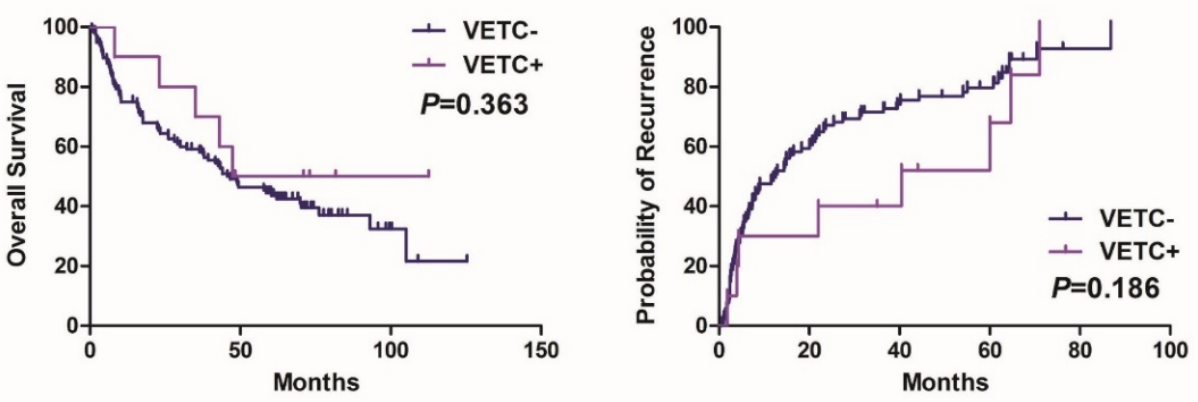

B
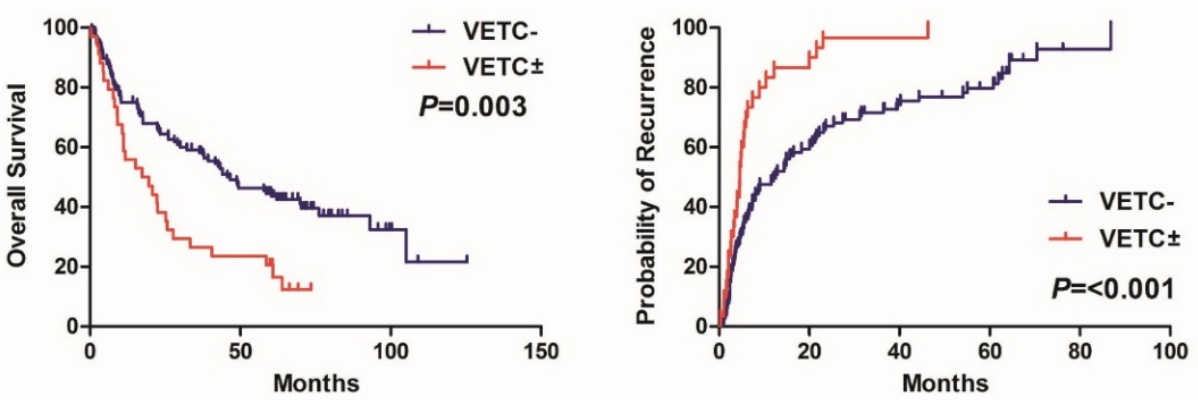

C
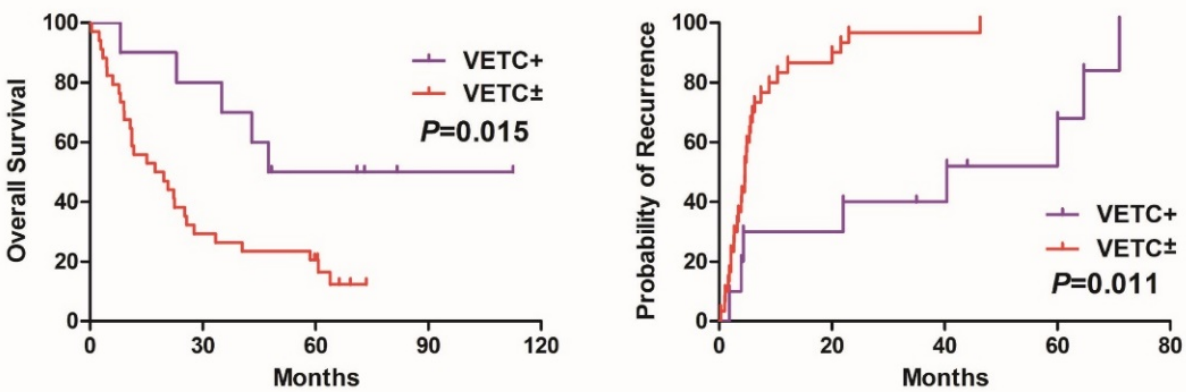

Figure 2. Kaplan-Meier curves of overall survival and time to recurrence were shown for patients with HCC. (A) HCC Patients with VETC- showed no differences in overall survival (left panel) and recurrence rates (right panel) compared with HCC patients with VETC+ ${ }^{+}$. $B$, C) The presence of VETC ${ }^{ \pm}$in HCC tissues was associated with shorter overall survival (left panel) and higher recurrence rates (right panel) compared with patients with (B) VETC- and (C) VETC+, respectively. 

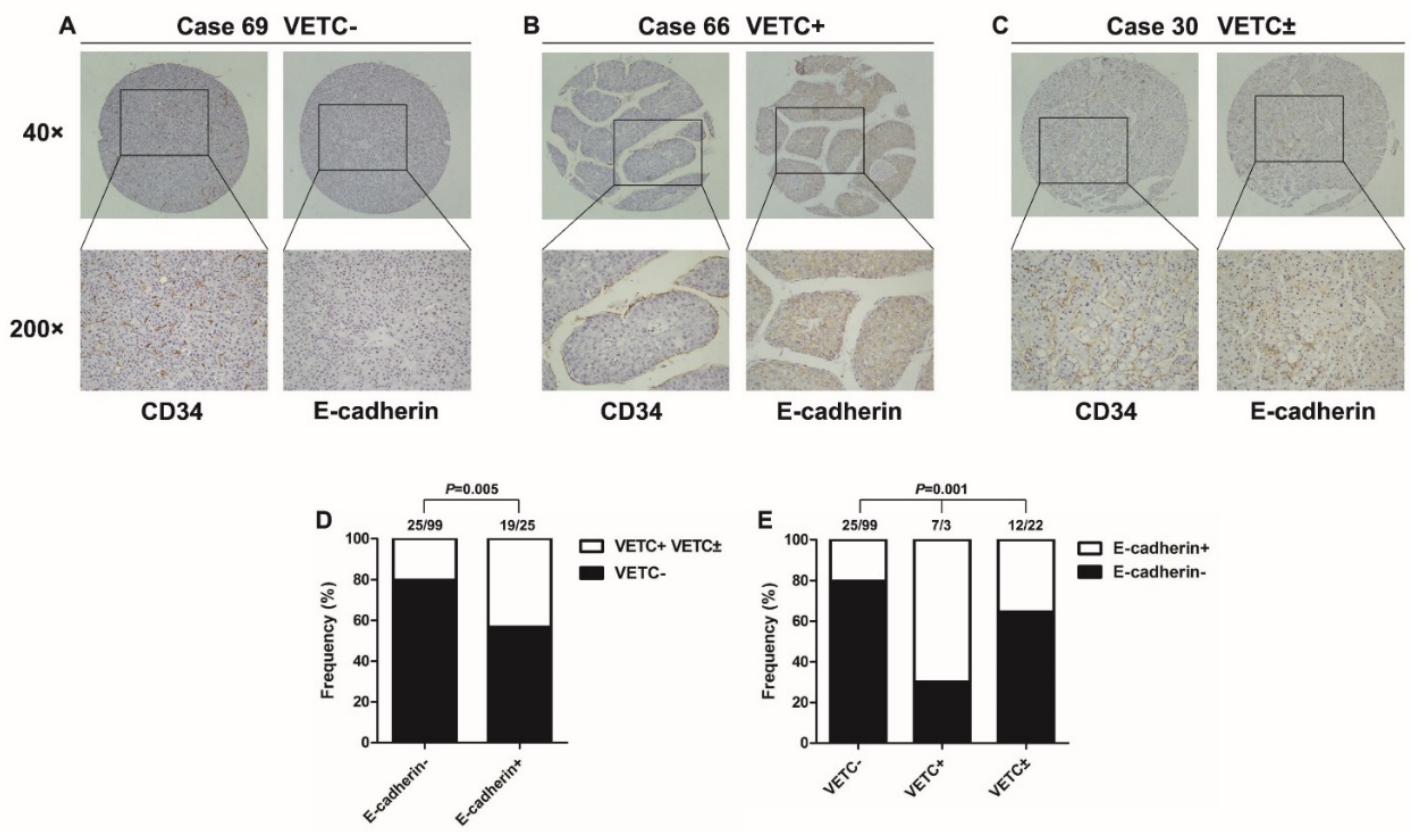

Figure 3. The presence of VETC structure was positively correlated with E-cadherin expression. (A) E-cadherin was lowly expressed in VETC- HCC tissues, and (B) highly expressed in VETC+ HCC tissues. (C) E-cadherin expression was highly expressed in VETC positive area, but lowly expressed in VETC negative area in VETC \pm HCC tissues. (D, E) High expression of E-cadherin was more frequently found in VETC-present HCC tissues (D), especially in HCC tissues of VETC+ group (E).

\section{The presence of VETC structure is positively correlated with E-cadherin expression but negatively correlated with snail expression}

E-cadherin is an important epithelial marker, and its functional loss is considered a hallmark of EMT [8]. To determine whether vascular pattern type is correlated with E-cadherin expression in HCC, we detected the expression of E-cadherin in the same tissue microarrays of $168 \mathrm{HCC}$ samples. E-cadherin expression was down-regulated in VETC- patients (Fig. 3A), and up-regulated in $\mathrm{VETC}^{+}$patients (Fig. 3B). Interestingly, in $\mathrm{VETC}^{ \pm}$group, E-cadherin expression was higher in VETC positive area, compared with VETC negative area of the same section (Fig. 3C). Inversely, the expression of snail was negatively correlated with the presence of VETC structure (Fig. S1). Statistical analysis showed that the presence of VETC structure was significantly associated with increased E-cadherin expression (Fig. 3D). In addition, there were significantly more patients with positive E-cadherin expression in $\mathrm{VETC}^{+}$group than in $\mathrm{VETC}^{ \pm}$group (Fig. 3E).

\section{EMT is the main manner for intrahepatic metastasis in VETC ${ }^{ \pm}$patients}

Previous studies have demonstrated that VETC could promote HCC metastasis in a VETC-mediated manner [15]. To investigate the main metastatic manner of HCC with VETC $^{ \pm}$, vascular pattern type was detected in primary and intrahepatic metastatic lesions of HCC. Overall, 50 pairs of HCC samples were examined. In primary lesions, 68\% (34/50) were VETC- (VETC- group), 10\% (5/50) were $\mathrm{VETC}^{+}$cases (VETC $^{+}$group), and $22 \%(11 / 50)$ were $\mathrm{VETC}^{ \pm}$cases (VETC ${ }^{ \pm}$group). The vascular pattern types of intrahepatic metastatic lesions of VETC- and VETC ${ }^{+}$ groups were consistent with their primary lesions (Fig. 4A, B, upper). However, in the 11 intrahepatic metastatic lesions of $\mathrm{VETC}^{ \pm}$group, nine $(81.8 \%)$ were VETC negative, and two (18.2\%) were VETC positive (Fig. 4C, upper panel; Fig. S2, upper panel). Moreover, the expression of E-cadherin was positively correlated with the presence of VETC structure (Fig. 4A-C, lower panel; Fig. S2, lower panel). These results suggested that EMT may be the main manner during intrahepatic metastasis in both VETC ${ }^{ \pm}$and VETCcases.

\section{EMT may be the main manner for major vascular invasion in VETC ${ }^{ \pm}$patients}

To further investigate the main metastatic manner of VETC $^{ \pm}$HCC, we examined the vascular pattern types in 12 paired HCC and mPVTT tissues. Among the 12 primary HCC tissues, seven were VETC- cases (VETC- group), five were VETC $^{ \pm}$cases (VETC ${ }^{ \pm}$group) (Fig. 5A, B, left). However, the vascular pattern types of mPVTTs in both groups were all VETC negative (Fig. 5A, B, right). These data suggested that tumor cells of HCC with $\mathrm{VETC}^{ \pm}$may invade into major vasculature through an EMT-dependent manner. 

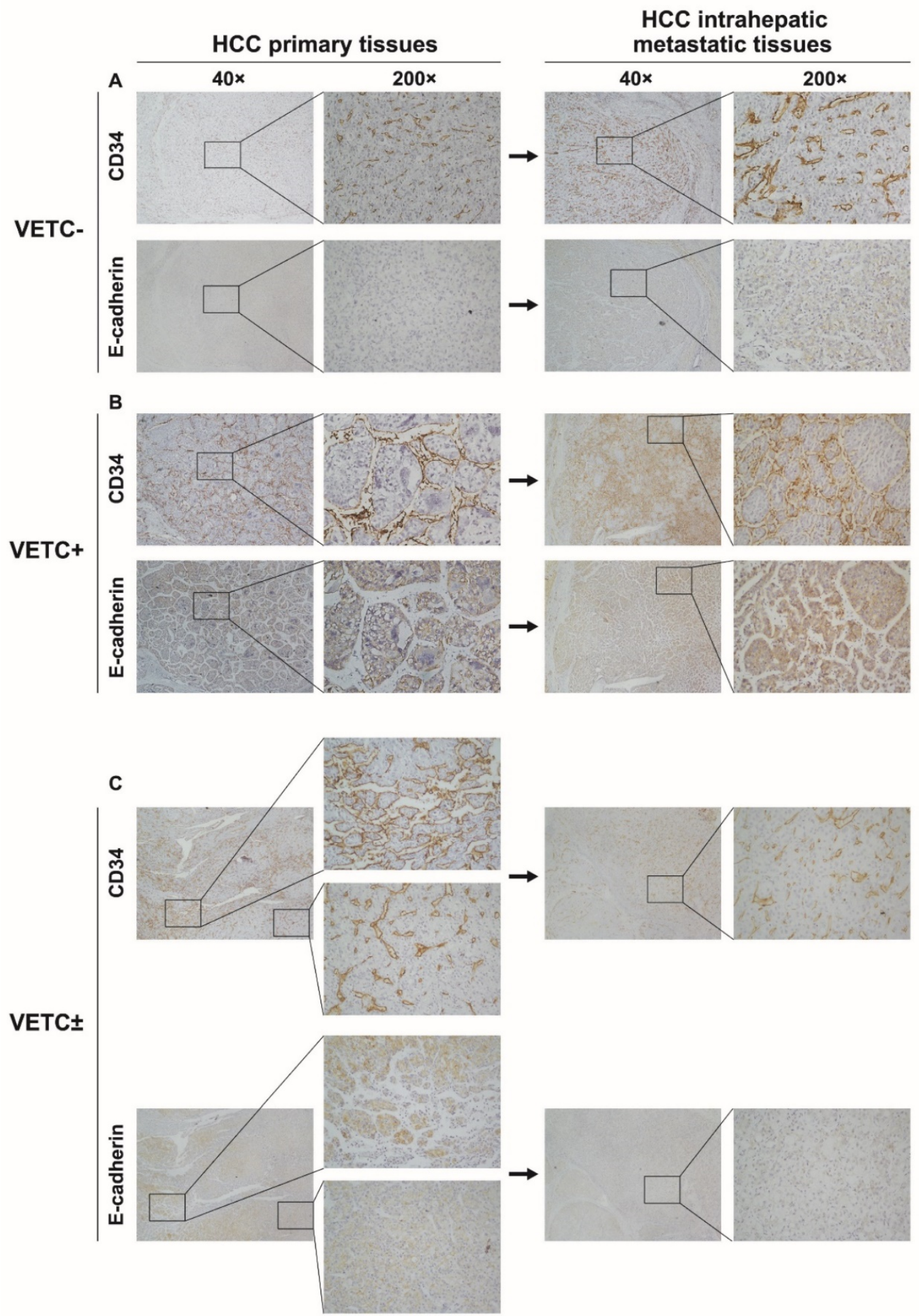

Figure 4. Vascular pattern type and E-cadherin expression in 50 paired primary and intrahepatic metastatic lesions. (A, B, upper panel) Vascular pattern types of intrahepatic metastatic lesions were similar to their corresponding primary lesions in (A) VETC-and (B) VETC ${ }^{+}$group. (C, upper panel) Intrahepatic metastatic lesions from VETC \pm group showed no VETCs. (A-C, lower panel) In serial sections, E-cadherin expression was highly expressed in VETC positive area, but lowly expressed in VETC negative area in HCC tissues. 

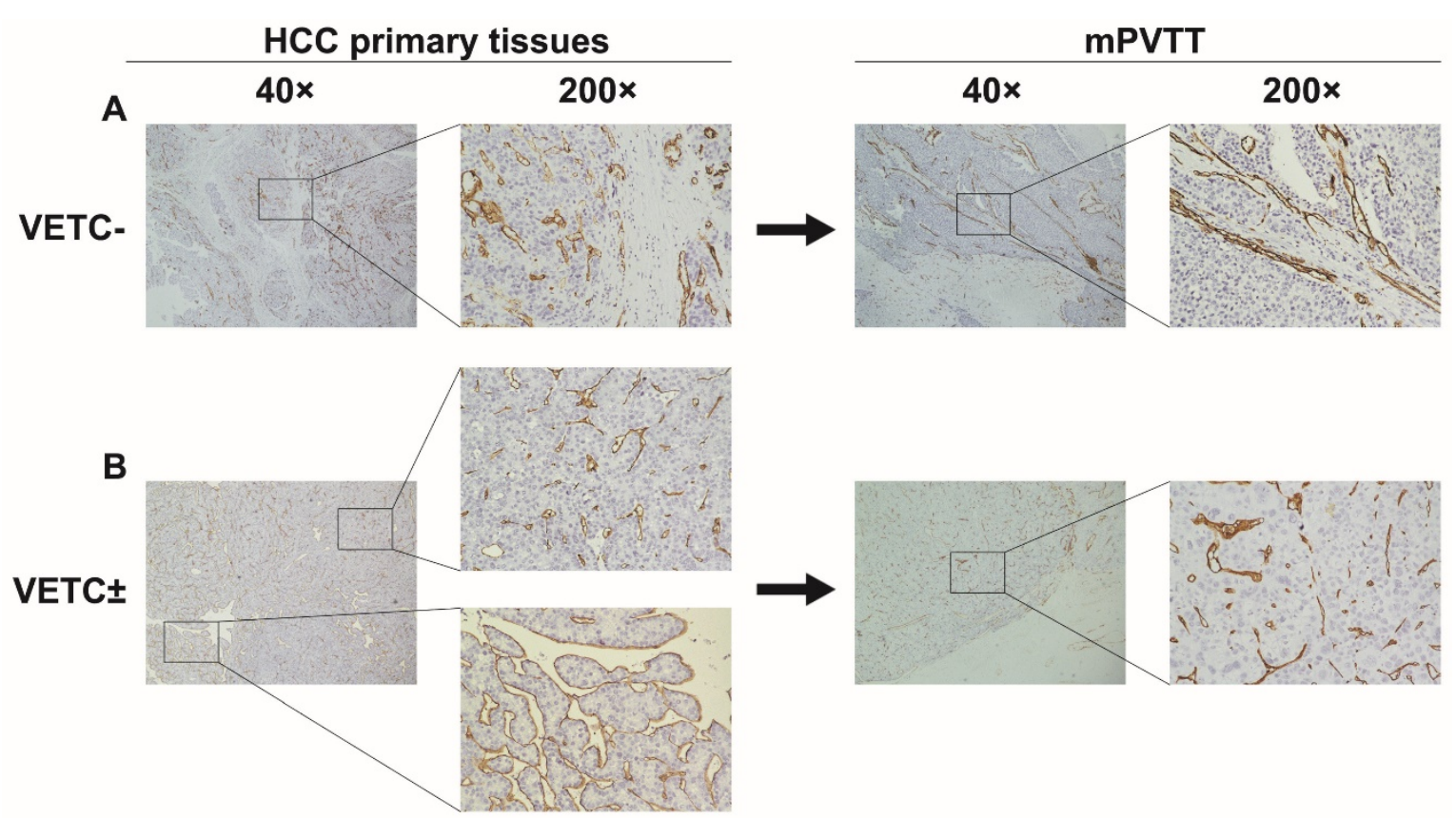

Figure 5. Vascular pattern type and E-cadherin expression in 12 paired HCC and mPVTT tissues. (A) The vascular pattern types of mPVTTs in VETC-group were consistent with their primary lesions. (B) The vascular pattern types of all mPVTT sections of VETC ${ }^{\mathrm{A}}$ group were VETC negative. Abbreviation: $\mathrm{mPVTT}$, major portal vein tumor thrombus.

\section{Discussion}

VETC is a peculiar vascular structure in which tumor cells are enveloped by endothelial cells to form tumor islands [13]. A previous study reported that VETC was observed in most cases of HCC, renal cell carcinomas, and follicular thyroid carcinomas, but was rarely seen in other seven kinds of cancer, such as lung and stomach cancer [16]. Later, a study demonstrated that VETC was commonly found in HCC (39\%), and the presence of VETC structure was an independent prognostic indicator of both shorter overall survival and higher recurrence rates [14]. Our data were consistent with these results. In our cohort of 168 HCC cases, VETC structure can be found in $26.2 \%$ of HCC cases. However, we found that in most of VETC-present HCC tissues, the VETC structure was distributed unevenly with capillary-like pattern. In order to elucidate whether this mixed vascular pattern was an important factor in determining clinical outcomes of HCC patients, we classified vascular pattern as VETC-, VETC $^{+}$, and VETC $^{ \pm}$ according to the proportion of VETC structure. Accordingly, the presence of $\mathrm{VETC}^{ \pm}$was more common than $\mathrm{VETC}^{+}(20.2 \%$ vs. $6 \%)$. Furthermore, Kaplan-Meier analysis showed that HCC patients with $\mathrm{VETC}^{ \pm}$had worse prognoses than those with VETC- or $\mathrm{VETC}^{+}$. Additionally, multivariate analysis revealed that the presence of $\mathrm{VETC}^{ \pm}$was an independent and significant risk factor for survival and recurrence of HCC after curative resection. Our clinical data strongly suggested that $\operatorname{VETC}^{ \pm}$may contribute to the progression of HCC.

A recent study demonstrated that VETC could promote metastasis of HCC in an EMT-independent but VETC-mediated manner [15]. They showed evidences that endothelium-coated tumor cell clusters were frequently found in livers and lungs of VETC-present HCC xenograft mice [15]. However, whether this phenomenon exists in human HCC tissue remains unknown. In this study, we found that the vascular pattern of both primary and intrahepatic metastatic lesions of $\mathrm{VETC}^{+}$group was VETC positive. However, our results also showed that when HCC tissues consisted of both VETC positive and VETC negative structure, the vascular pattern of their metastases (both intrahepatic and major vascular) was more likely to be VETC negative. Thus, our results suggested that HCC containing both VETC and capillary-like structure may prefer to metastasize in a VETC-independent manner.

Our results showed that the presence of VETC structure was positively correlated with E-cadherin expression, even in the same HCC tissue section. Interestingly, statistical results showed that in 168 HCC cases, VETC ${ }^{ \pm}$group has lower proportion of E-cadherin-positive cases than $\mathrm{VETC}^{+}$group $(35.3 \%$ vs. 70\%). The main reason for these results is that most cases of $\mathrm{VETC}^{ \pm}$group contained a small area of VETC structure, which were corresponding to a low extent of E-cadherin staining or even deficient expression of E-cadherin. E-cadherin is a key bio-marker for EMT 
[8]. Almost all the EMT-inducing transcription factors are reported to be capable of repressing E-cadherin expression, which are considered to be critical events during EMT [21]. Thus, our results implied that HCC with VETC ${ }^{ \pm}$may metastasize potentially through an EMT-dependent manner. Consistent with this, we found that E-cadherin was down-regulated in metastatic lesions of not only VETC- group, but also VETC $^{ \pm}$group. In addition, our data also showed that the expression of snail, one of the key transcription factors during EMT [8], was negatively correlated with the presence of VETC structure in 168 HCC cases. These findings indicated that EMT may be the main manner for HCC with $\mathrm{VETC}^{ \pm}$to metastasize.

Personalized treatment according to the presence of molecular targets will improve the outcomes for patients with malignant diseases [22]. To date, many agents have been reported to be capable of inhibiting EMT [23]. On the other hand, Angiopoietin 2 (Ang2) has been proved to be a critical determinant in VETC formation as well as VETC-mediated metastasis, suggesting that anti-Ang2 treatment may be beneficial for VETC-present patients [15]. However, our data indicated that in cases of HCC with VETC ${ }^{ \pm}$, both anti-EMT and anti-VETC agents should be considered because the tumor cells may probably metastasize via an EMT-dependent manner.

In this study, we make our conclusions only through immunohistochemical findings. However, more studies are required to elucidate the molecular mechanism how $\mathrm{VETC}^{ \pm}$promotes metastasis in HCC. Despite this limitation, our study indicates that the presence of $\mathrm{VETC}^{ \pm}$in $\mathrm{HCC}$ is a strong prognostic predictor of poor clinical outcome. HCC with VETC ${ }^{ \pm}$ may be more likely to metastasize in a VETC-independent but EMT-dependent manner, which suggests that EMT may be superior to VETC in promoting $\mathrm{VETC}^{ \pm}$cases metastasis. Our data also provide a clue that both anti-EMT and anti-VETC agents should be considered in the case of HCC with $V_{E T C}{ }^{ \pm}$. Hopefully, our results may provide a clue for developing personalized medicine for HCC patients.

\section{Supplementary Material}

Supplementary figures.

http://www.jcancer.org/v08p0039s1.pdf

\section{Acknowledgements}

This work was supported by grants from the National Natural Science Foundation of China (Nos. grant nos. 81301768, 81372565); Grant [2013]163 from Key Laboratory of Malignant Tumor Molecular Mechanism and Translational Medicine of Guangzhou Bureau of Science and Information Technology; Grant KLB09001 from the Key
Laboratory of Malignant Tumor Gene Regulation and Target Therapy of Guangdong Higher Education Institutes; Grant from Guangdong Science and Technology Department (2015B050501004). The authors would like to thank Professor Shimei Zhuang and Limin Zheng (School of Life Sciences, Sun Yat-Sen University) for their technical assistance, Doctor Qiaodong $\mathrm{Xu}$ for the clinicopathologic data review.

\section{Competing Interests}

The authors declare that they have no competing interests.

\section{References}

1 Llovet JM, Villanueva A, Lachenmayer A, et al. Advances in targeted therapies for hepatocellular carcinoma in the genomic era. Nat Rev Clin Oncol. 2015; 12: 408-424.

2 Liu PH, Hsu CY, Hsia CY, et al. Surgical Resection Versus Radiofrequency Ablation for Single Hepatocellular Carcinoma $</=2 \mathrm{~cm}$ in a Propensity Score Model. Ann Surg. 2016; 263: 538-545.

3 Renner P, Schuhbaum J, Kroemer A, et al. Morbidity of hepatic resection for intermediate and advanced hepatocellular carcinoma. Langenbecks Arch Surg. 2016; 401: 43-53.

4 Ryu SH, Jang MK, Kim WJ, et al. Metastatic tumor antigen in hepatocellular carcinoma: golden roads toward personalized medicine. Cancer Metastasis Rev. 2014; 33: 965-980.

5 Talmadge JE, Fidler IJ. AACR centennial series: the biology of cancer metastasis: historical perspective. Cancer Res. 2010; 70: 5649-5669.

6 Hanahan D, Weinberg RA. Hallmarks of cancer: the next generation. Cell. 2011; 144: 646-674.

7 Friedl P, Wolf K. Plasticity of cell migration: a multiscale tuning model. J Cell Biol. 2010; 188: 11-19.

8 Gonzalez DM, Medici D. Signaling mechanisms of the epithelial-mesenchymal transition. Sci Signal. 2014; 7: re8. doi: 10.1126/scisignal.2005189.

9 Tam WL, Weinberg RA. The epigenetics of epithelial-mesenchymal plasticity in cancer. Nat Med. 2013; 19: 1438-1449.

10 Chiang SP, Cabrera RM, Segall JE. Tumor Cell Intravasation. A Review in the Theme: Cell and Molecular Processes in Cancer Metastasis. Am J Physiol Cell Physiol. 2016; doi: 10.1152/ajpcell.00238.2015.

11 Harney AS, Arwert EN, Entenberg D, et al. Real-Time Imaging Reveals Local, Transient Vascular Permeability, and Tumor Cell Intravasation Stimulated by TIE2hi Macrophage-Derived VEGFA. Cancer Discov. 2015; 5: 932-943.

12 Pecot CV, Rupaimoole R, Yang D, et al. Tumour angiogenesis regulation by the miR-200 family. Nat Commun. 2013; 4:2427. doi: 10.1038/ncomms3427.

13 Sugino T, Yamaguchi T, Hoshi N, et al. Sinusoidal tumor angiogenesis is a key component in hepatocellular carcinoma metastasis. Clin Exp Metastasis. 2008; 25: 835-841.

14 Ding T, Xu J, Zhang Y, et al. Endothelium-coated tumor clusters are associated with poor prognosis and micrometastasis of hepatocellular carcinoma after resection. Cancer. 2011; 117: 4878-4889.

15 Fang JH, Zhou HC, Zhang C, et al. A novel vascular pattern promotes metastasis of hepatocellular carcinoma in an epithelial-mesenchymal transition-independent manner. Hepatology. 2015; 62: 452-465.

16 Sugino T, Yamaguchi T, Ogura G, et al. Morphological evidence for an invasion-independent metastasis pathway exists in multiple human cancers. BMC Med. 2004; 2: 9

17 Jia D, Jing $Y$, Zhang Z, et al. Amplification of MPZL1/PZR promotes tumor cell migration through Src-mediated phosphorylation of cortactin in hepatocellular carcinoma. Cell Res. 2014; 24: 204-217.

18 Shimada M, Hamatsu T, Yamashita Y, et al. Characteristics of multicentric hepatocellular carcinomas: comparison with intrahepatic metastasis. World J Surg. 2001; 25: 991-995.

$19 \mathrm{He} \mathrm{C}, \mathrm{Xu} \mathrm{J}$, Zhang J, et al. High expression of trimethylated histone H3 lysine 4 is associated with poor prognosis in hepatocellular carcinoma. Hum Pathol. 2012; 43: 1425-1435.

20 Mao K, Zhang J, He C, et al. Restoration of miR-193b sensitizes Hepatitis B virus-associated hepatocellular carcinoma to sorafenib. Cancer Lett. 2014; 352: 245-252.

21 Lamouille S, Xu J, Derynck R. Molecular mechanisms of epithelial-mesenchymal transition. Nat Rev Mol Cell Biol. 2014; 15: 178-196.

22 Hiley C, de Bruin EC, McGranahan N, et al. Deciphering intratumor heterogeneity and temporal acquisition of driver events to refine precision medicine. Genome Biol. 2014; 15: 453.

23 Marcucci F, Stassi G, De Maria R. Epithelial-mesenchymal transition: a new target in anticancer drug discovery. Nat Rev Drug Discov. 2016; 15: 311-325. 